\title{
Controller Performance Analysis Technology for Industry: Implementation and Case Studies *
}

\author{
Kwan Ho Lee*, Fangwei Xu**, Biao Huang ${ }^{\dagger *}$, \\ Edgar C. Tamayo ${ }^{* *}$ \\ * Chemical and Materials Engineering, University of Alberta, \\ Edmonton, Alberta T6G 2G6, Canada \\ ** Syncrude Canada Ltd., Fort McMurray, Alberta T9H 3L1, Canada
}

\begin{abstract}
In this paper, an industrial MPC performance monitoring technology is introduced with a focus on the industrial implementation. A plant-oriented framework for APC performance monitoring is proposed on the basis of industrial computer control systems background. A software package integrating this technology, which is called Performance Analysis Toolbox and Solutions (PATS), is introduced. The major components of PATS are discussed including process data collection, data preprocessing, process model identification, similarity clustering, control valve stiction detection, multivariate controller performance assessment, and APC economic performance assessment using linear matrix inequality optimization. An industrial case study of a hydrogen unit is illustrated. A limited trial version of the software package can be downloaded from the web http://www.ualberta.ca/ bhuang/research/research.htm
\end{abstract}

\section{INTRODUCTION}

A controller performance monitoring technology, which is called Performance Analysis Toolbox and Solutions (PATS), has been developed with collaborations between academia and process industries. Many algorithms have been integrated into the software package. It includes a number of independent components and each of them has its own initiative from industries. All components of this software package can be classified into three categories in terms of overall functionality, namely, interface components, assistant components and application components as shown in Fig. (a) 1. The interface components were designed for retrieving raw process data from different sources, such as data historian with historical data (1 component), OPC (OLE for Process Control, where OLE is referred to as Object Linking and Embedding technique) server with real-time data (2 components), and virtual plants with simulated data (1 component). The assistant components are used to deal with these raw data and provide the application components with consistent and cleaned data. Some routine work of these assistant components includes data preparation, data preprocessing, etc. The main task of process data analysis is carried out by application components with different design objectives driven by process industries. They cover the most state-ofthe-art technologies which have been transferred directly from recent research work in the fields of controller performance monitoring ( 3 components), process model identification (2 components), fault detection and isolation (1 component), and controller design (1 component). PATS bridges the gap between academia and industries, and

\footnotetext{
* The work is supported by Syncrude Canada Ltd. and the Natural Sciences and Engineering Research Council of Canada (NSERC).

${ }^{\dagger}$ Corresponding author Biao Huang. E-mail: biao.huang@ualberta.ca
}

makes it possible to apply the research outcome directly and rapidly into process industries.

One of the main purposes of PATS is to perform controller performance monitoring in process industries, especially for the widely used model predictive control (MPC) applications. MPC has been proven as one of the most effective advanced process control (APC) strategies to deal with multivariable process systems with input/output constraints [Qin and Badgwell, 2003]. Even though the rewards of MPC applications can be great, some of them may not be used to their full capacity in practice. Less effort has been made on the performance monitoring of existing industrial MPC applications, especially on the economic performance. Therefore, a systematic and standardized approach is demanded by the process industries to facilitate the task of MPC maintenance.

In this paper, an implementation background is introduced in Section 2. A plant-oriented framework for APC performance monitoring is proposed in Section 3. A solutions package, called PATS, is introduced in Section 4. An industrial case study of a hydrogen unit is illustrated using PATS in Section 5 followed by concluding remarks in Section 6.

\section{IMPLEMENTATION BACKGROUND FOR APC PERFORMANCE MONITORING}

Our industrial practice and experience for APC performance monitoring will be introduced in this section. The software package PATS has been tested and implemented in a process industry, particularly for APC performance monitoring. To this date there are many MPC applications implemented in different plants of the industry. Unfortunately, it is rather hard for process control engineers to routinely monitor the performance of the run-time MPC 


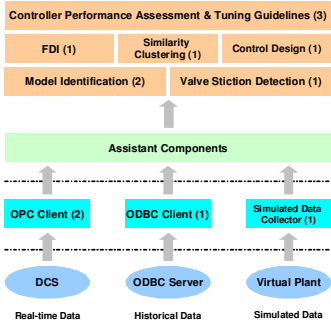

(a)

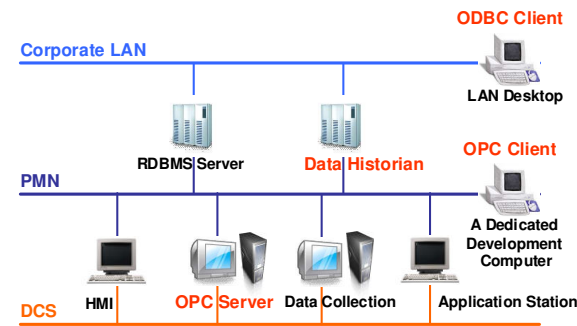

(b)

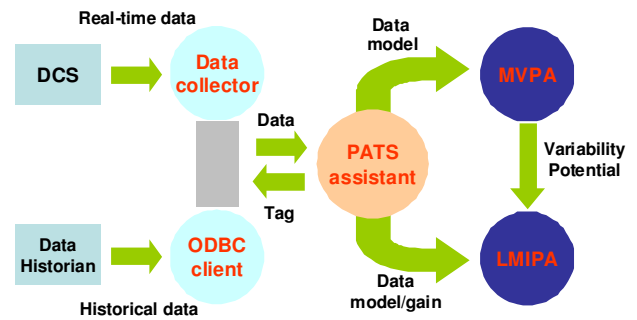

(c)

Fig. 1. (a) An overview of the software package PATS. (b) A computer control systems structure in industries. (c) A plant-oriented framework for APC performance monitoring.

applications. By the implementation of PATS, a practical plant-oriented solution has been carried out for APC performance monitoring, which is based directly on the existing distributed control systems (DCS). It tells not only the output variance performance but also the economic performance and MPC tuning guidelines, by taking full advantage of existing available process data information.

The overall computer control system structure is illustrated in Fig. (b) 1. There are three network layers, including DCS network at the bottom, process management network (PMN) in the middle and corporate local area network (LAN) on the top. The distributed controllers running regulatory control loops are all connected to the DCS network via network interfaces. The system components are human-machine interface (HMI), application station, OPC server and data collection between DCS and PMN and relational database management system (RDBMS) server and data historian between PMN and LAN. The APC applications are implemented and running on the application station. The real-time data can be collected from the OPC server via OPC client. The data collection samples the real-time data from DCS with the background supported by the RDBMS server and at the same time transfers the collected data to the data historian in a batch mode. The data historian keeps the historical data information which can be accessed from any corporate LAN desktop computers.

PATS includes two interface components, one is an OPC client, which is also named real-time data collector, and the other one is ODBC client, where ODBC stands for open database connectivity protocol. PATS can be loaded on any LAN desktop computer with an ODBC client as the data interface and data historian as the data source. In this way the historical data can be retrieved for analysis. For the real-time data, the OPC client is installed on a dedicated development computer, which has a direct connection with PMN such that the OPC client can have access to the OPC server. Therefore, PATS can also be implemented for real-time data analysis. It should be mentioned that this framework is built on the basis of existing control systems infrastructure, but it is readily transplanted to and integrated with other control systems.

\section{A PLANT-ORIENTED FRAMEWORK FOR APC PERFORMANCE MONITORING}

As shown in Fig. (c) 1, a plant-oriented framework is proposed with the implementation background in Fig. (b) 1, especially for APC performance monitoring. It is composed of two main application components, two interface components and some assistant components of the PATS package.

The historical process data can be retrieved from the data historian by an ODBC client and the real-time process data from the DCS by real-time data collector via OPC technique. Both of the historical data and real-time data are then transferred and supplied by the assistant components to the application components. The assistant components serve as a process information bridge or hub between the interface components and the application components. On the one hand, they provide convenient utilities to enter process tag names for the interface components and process model information for the application components if it is required. On the other hand, the collected process data are preprocessed, such as detecting and removing outliers, and then standardized in a format such that the data set is consistent with the application components.

\section{PERFORMANCE ANALYSIS TOOLBOX AND SOLUTIONS}

\subsection{Process data collector}

There are three components for process data collection. The first one is Real-time Data Collector, which collects real-time data from an OPC server. Real-time Data Collector is provided with an additional component, namely, Tag Generator. In APC applications, there are a number of controlled variables (CV), manipulated variables (MV) and disturbance variables (DV), and each is associated a number of parameters such as low/high limit, soft low/high limit, on/off status, process value, etc. Manually entering all these tags is a tedious and time consuming job. This tool helps in generating the tag list for all the CVs, MVs and DVs. Once the tag list has been created using Tag Generator, it can be loaded into Real-time Data Collector, and the data collection can be started. The second one is History Data Collector, which saves plant history data from ODBC server. It is also provided with Tag Generator. The third one is Simulink Data Collector. This is a tool which can be used, if the plant model is known, to build an APC application, to run the simulation for data generation, in the required format, for carrying out the further analysis of the data. The data created in the components can be saved in several file formats. Fig. (a) 2 shows Real-time Data Collector, as an example. 


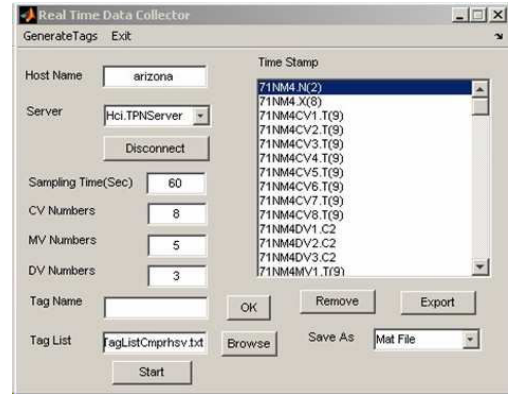

(a)

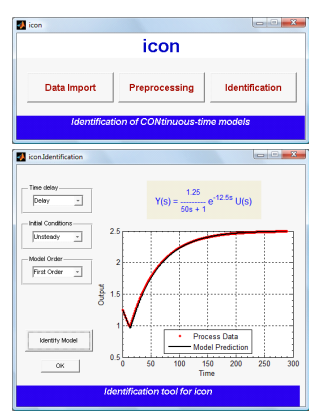

(b)

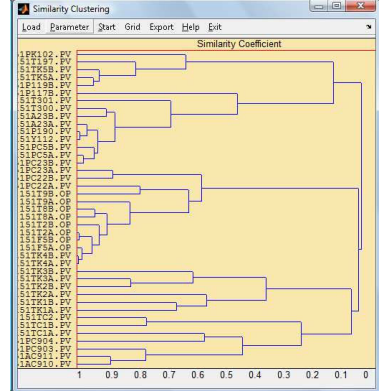

(c)

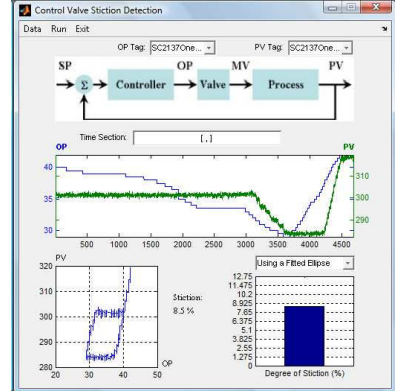

(d)

Fig. 2. (a) Process data collection tool: Real-Time Data Collector. (b) Model identification tool: ICON. (c) Similarity clustering tool. (d) Control valve stiction detection tool.

\subsection{Data pre-processing}

The data created either in Real-time Data Collector, History Data Collector, or Simulink Data Collector, is in a raw data format. It needs to be converted into a specific format, in order to enable it to be analyzed by the application components. Also the raw data may contain some outliers that need to be removed from the data set. This tool facilitates the deletion of data points for the time stamp where there is at least one outlier.

\subsection{Model identification}

The process model identification tools can be used for identifying the process model transfer function using process input/output data. Fig. (b) 2 shows the GUI for model identification. This component makes use of a continuous time step response method, a subspace method and a prediction error method to identify the process with appropriate process data. For the continuous time step response method, the imported process data should be step response data. For the subspace method and prediction error methods, the input to the process should be input perturbations with sufficient excitation. This tool can generate $1^{\text {st }}$-order or $2^{\text {nd }}$-order continuous time transfer function models. The simulated plots will be shown in the same figure with the actual time plots for comparison. In performing controller performance assessment, it is required to have a process model in the required format. This tool is provided with an additional component, namely, Model Generator, which helps in generating a model file in the required format. Fig. (b) 2 shows the GUI of the process model identification tool, called ICON (identification of continuous-time models).

\subsection{Similarity clustering}

The fundamental principle of cluster analysis is the grouping of multivariate data, in which numerous correlations between data tags exist. Cluster algorithms are a generic tool for pattern recognition, the search for structure in data, with a wide range of applications. As an application in the process industry, the similarity clustering tool is used to group process variables according to their correlations by taking into account time delay effects. It can be used to analyze causal relations for some process phenomena, for example, to detect the source of fault or breakdown. The similarity clustering algorithm consists of two essential parts: the calculation of similarity coefficients and the clustering of variables. The first part is to calculate the similarity coefficient between two variables. The second part of the algorithm is to use the Agglomerative Hierarchical method with Complete Linkage to cluster the variables, based on the similarity matrix obtained in the first part. The clustering starts from the individual variables. the most similar variables are first grouped, and these initial groups are merged according to their similarities. Groups are fused according to the similarity between their farthest members (the two variables with smallest similarity coefficient) so that all variables in a cluster are within some minimum similarity of each other. The results of the clustering procedure is displayed on the GUI in the form of a two dimensional diagram known as a dendrogram. The level of each group or subgroup represents the value of similarity coefficient, a value that every two variables in that group are ensured to be larger than it. Fig. (c) 2 shows the GUI of the similarity clustering tool. The distinguishing feature of this tool in PATS is its ability to search for an optimal time delay before grouping.

\subsection{Valve stiction detection}

A basic control system with a valve is depicted in Fig. (d) 2 , where $\mathrm{SP}, \mathrm{OP}, \mathrm{MV}$, are PV represent set point, control signal, manipulated and controlled variables, respectively. In general, SP, OP and PV are recorded in the DCS systems, and so are readily available, but the real value of a manipulated variable MV such as flow rate is not available in most control loops. The valve stiction can be represented as the necessary force applied to the valve stem to make it move. The valve will not move if the amount of force corresponding to the controller output is too small to overcome the static friction. The controller may cause OP to increase in the same direction until the valve overcomes the stiction. Then, the valve moves suddenly with more than the desired amount causing the process to overshoot. Then OP changes in the opposite direction to get the process back on track until the valve overcomes the stiction, which makes the process overshoot again in the opposite direction, thus causing process oscillation. This tool estimates the extent of stiction in control loops. It performs the filtering of the control valve PV and OP values and then plots PV vs. OP data to observe hysterisis. The presence of hysterisis indicates stiction. This tool adopts several algorithms to quantify the degree of stiction. 


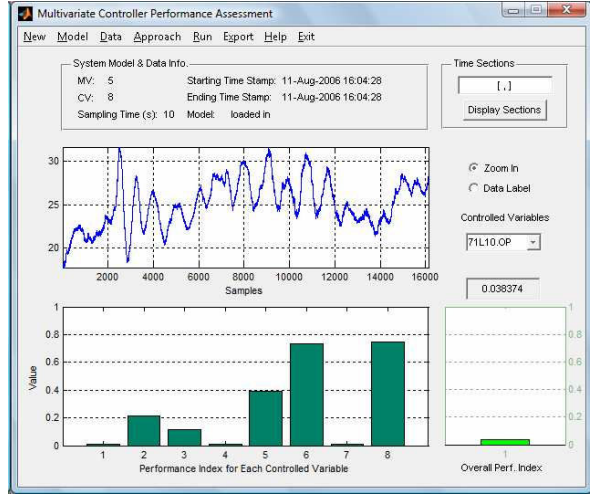

(a)

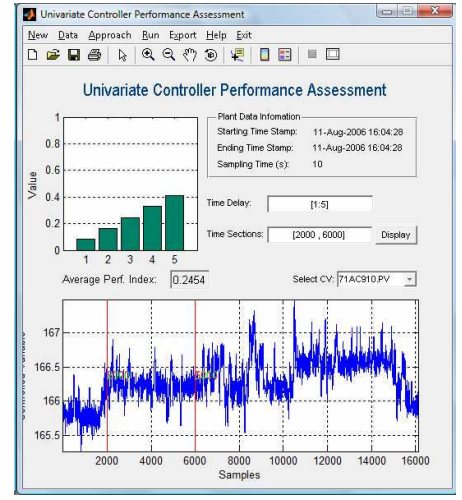

(b)

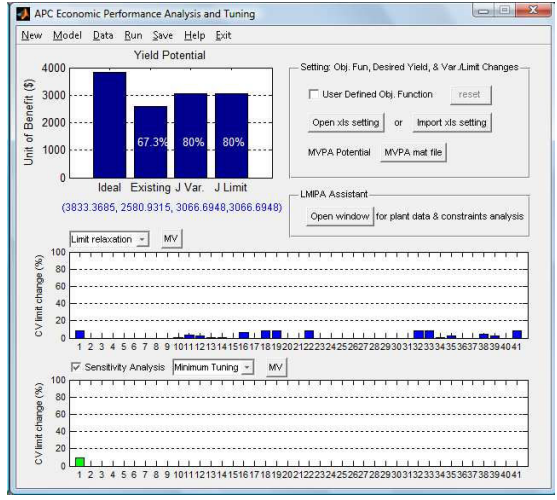

(c)

Fig. 3. (a) Multivariate performance assessment tool: MVPA. (b) Univariate performance assessment tool: UVPA. (c) APC economic performance assessment tool: LMIPA.

\subsection{Multivariate controller performance assessment}

There are thousands of control loops in process industries, which range from PID control loops to advanced multivariable control loops. Although those control loops are initially well tuned, their performance can gradually degrade due to change of process dynamics, wear of instruments and time varying disturbances. A major task is to effectively monitor the performance of control loops and to determine when and whether control loops need to be re-tuned, instruments need to be replaced or processes need to be re-engineered. How to evaluate multivariable control loops is becoming one of the most important tasks in process control applications.

MVPA is referred to as multivariate controller performance assessment component with minimum variance control (MVC) as the benchmark. It employs an FCOR algorithm to calculate the individual and overall variance performance indices [Huang and Shah, 1999]. The variance performance indices quantify how good the existing control loop is compared with the MVC benchmark, and gives rise to the improvement potential in the sense of output variance. Fig. (a) 3 shows the GUI of MVPA.

The procedure of MVPA is divided into two major steps. The first step is to calculate the unitary interactor matrix from the open-loop process model. The second step is to calculate performance indices by doing time series analysis from the closed-loop routine operating data. For the detailed algorithms, refer to [Huang and Shah, 1999]. The input of this component includes a process model and closed-loop routine operating data. MVPA shows a general performance index as well as performance indices of the different control variables. Using the general performance index or performance indices of the different control variables, process control engineers can estimate the potential to improve the performance of current multivariable control loops. If the performance is lower than the expected, the monitor can provide timely warning for the process control engineers such that corresponding actions are taken to improve the performance, such as re-tuning control parameters or revising model parameters. MVPA is a tool that can be utilized to measure the performance of the multivariable control loops from a variability point of view.
For performance assessment of univariate control loops, PATS also provides a separate tool called UVPA as shown in Fig. (b) 3, which stands for univariate controller performance assessment. It identifies a time series model from routine operating process data and estimates a variability performance index for single loops. For the detailed algorithms, see [Huang and Shah, 1999].

\subsection{APC economic performance assessment}

LMIPA stands for a linear matrix inequality approach for performance assessment with an objective for providing APC economic performance assessment and tuning guidelines. It takes a process steady-state gain, process input/output data, and relevant control parameters such as input/output constraint limits and then calculates benefit potentials and economic performance indices for different scenarios which can be considered in practice. Also it suggests MPC constraint and variability tuning guidelines for economic benefit improvement. The variability improvement potential from the MVPA component can be provided to the LMIPA component for a theoretical benefit potential estimation with minimum variance control as the benchmark. This bridges the MVPA component and the LMIPA component within this plant-oriented solution. The GUI is shown in Fig. (c) 3. The algorithms for MPC economic performance assessment and tuning guidelines have been introduced in [Xu et al., 2007]. Also, this APC performance analysis tool includes a sensitivity analysis, which provides a selective constraint/variability tuning guidelines for benefit improvement in practice [Lee et al., 2007]. In the following, the main concepts for LMIPA will be summarized.

For a $p \times m$ process $G$, which is controlled by a model predictive controller, with $m$ input variables and $p$ output variables and a steady-state process gain matrix $K$, we define the current mean operating point for $\mathrm{i}^{\text {th }}$ output and $\mathrm{j}^{\text {th }}$ input variable as $\left(\bar{y}_{0 i}, \bar{u}_{0 j}\right)$, which is referred to as the base case operating point in this paper. Also, define the low and high limit of $y_{i}$ as $L_{y i}$ and $H_{y i}$, respectively, and the low and high limit of $u_{j}$ as $L_{u j}$ and $H_{u j}$, respectively. For an operating point $\left(\bar{y}_{i}, \bar{u}_{j}\right)$ of the process $G$, the economic objective function for the process is given by a quadratic function of the form: 


$$
\begin{aligned}
J=\sum_{i=1}^{p}\left[b_{i} \times \bar{y}_{i}+a_{i}^{2}\left(\bar{y}_{i}-\mu_{i}\right)^{2}\right] \\
+\sum_{j=1}^{m}\left[b_{j} \times \bar{u}_{j}+a_{j}^{2}\left(\bar{u}_{j}-\nu_{j}\right)^{2}\right]
\end{aligned}
$$

where $b_{i}$ and $a_{i}$ are the linear and quadratic coefficients for the controlled variable $y_{i}, b_{j}$ and $a_{j}$ are the linear and quadratic coefficients for the manipulated variable $u_{j}$, and $\mu_{i}$ and $\nu_{j}$ are the target values for $\mathrm{i}^{\text {th }} \mathrm{CV}$ and $\mathrm{j}^{\text {th }} \mathrm{MV}$, respectively. Note Eqn. (1) is a cost function. The optimal operation is to minimize it.

For the base case operation, the economic objective function value is readily calculated by replacing $\left(\bar{y}_{i}, \bar{u}_{j}\right)$ with the current operating point $\left(\bar{y}_{0 i}, \bar{u}_{0 j}\right)$ in (1). This objective value is denoted as $J_{0}$, which is a value to be used in the calculation of benefit potentials as a reference.

We perform assessment of yield for various cases described in detail below:

(1) Assessment of ideal benefit potential

(2) Assessment of existing benefit potential

(3) Assessment of improved yield by variability reduction

(4) Assessment of improved yield by constraint relaxation

(5) Constraint/variability tuning for a desired yield

Assessment of ideal benefit potential What would be the maximum benefit achieved if process variability could be reduced to zero? This problem is answered by the ideal benefit potential. For assessing the ideal yield, steady state operations are considered with no variability in both $y_{i}$ and $u_{j}$. Under this scenario the optimization problem for the system is defined as:

$$
\operatorname{Minimize}_{\left\{\bar{y}_{i}, \bar{u}_{j}\right\} \quad J}
$$

subject to:

$$
\begin{gathered}
\Delta y_{i}=\sum_{j=1}^{n} K_{i j} \times \Delta u_{j} \\
\bar{y}_{i}=\bar{y}_{0 i}+\Delta y_{i} \\
\bar{u}_{j}=\bar{u}_{0 j}+\Delta u_{j} \\
L_{y i} \leq \bar{y}_{i} \leq H_{y i}, \quad \forall i=1,2, \ldots, p \\
L_{u j} \leq \bar{u}_{j} \leq H_{u j}, \quad \forall j=1,2, \ldots, m
\end{gathered}
$$

Note that the present disturbance is not considered in the ideal benefit potential calculation. The solution of the problem gives rise to an ideal optimal operating condition and the corresponding objective function is denoted as $J_{I}$. The ideal benefit potential $\Delta J_{I}$ is given by $\Delta J_{I}=J_{0}-J_{I}$.

Assessment of existing benefit potential What benefit can be achieved by simply adjusting the operating points without changing any constraint or reducing the variability? This problem is answered by the existing benefit potential. The assessment of the optimal yield of the controller without tuning means to assess the yield that should be obtained from the controller for the given constraints and the existing variability in base case operations. This scenario considers moving the actual operating point of $y_{i}$ to its optimal operating conditions, as close as possible, subject to the constraints. Under this scenario the optimization problem for the process is to solve (2) subject to (3)-(5) and

$$
\begin{gathered}
L_{y i}+2 \times \sigma_{0 i} \leq \bar{y}_{i} \leq H_{y i}-2 \times \sigma_{0 i} \\
L_{u j}+2 \times R_{0 j} \leq \bar{u}_{j} \leq H_{u j}-2 \times R_{0 j}
\end{gathered}
$$

where $\sigma_{0 i}$ and $R_{0 j}$ are the base case standard deviation and the quarter of the range for $y_{i}$ and $u_{j}$, respectively. If the constraints (8)-(9) are infeasible, then replace (8) (resp. (9)) by

$$
\begin{gathered}
\bar{y}_{i}=\bar{y}_{0 i} \quad \text { or } \quad \Delta \bar{y}_{i}=0 \\
\left(\text { resp. } \quad \bar{u}_{i}=\bar{u}_{0 i} \quad \text { or } \quad \Delta \bar{u}_{j}=0\right)
\end{gathered}
$$

The inequalities defined above allow $5 \%$ constraint limit violation, i.e., $95 \%$ of the operation is within two standard deviations [Latour et al., 1986, Martin et al., 1991]. Note that the present disturbances are taken into account in the existing benefit potential calculation and the corresponding objective function is denoted as $J_{E}$. The existing benefit potential $\Delta J_{E}$ is given by $\Delta J_{E}=J_{0}-J_{E}$.

Assessment of improved yield by reducing variability This case involves tuning of the control system such that the variability of one or more output variables can be reduced. Reducing the variability provides an opportunity to push the operating points closer to the optimum and thus improve the yield. Practically, the reduction in variance of one variable (say quality variable) is often transferred to the variability of some other variables, such as constrained variables. Since constraint variables do not directly affect the profit, their variability is not of concern, as long as they are maintained within the set limits. Thus, variability of a quality variable can be reduced by transferring it to that of the constraint variables. For assessing the improved yield by variability reduction the optimization problem is to solve (2) subject to (3)-(5) and (12)-(13)

$$
\begin{aligned}
L_{y i}+2 \times \sigma_{0 i} \times & \left(1-S_{y i}\right) \\
& \leq \bar{y}_{i} \leq H_{y i}-2 \times \sigma_{0 i} \times\left(1-S_{y i}\right) \\
L_{u j}+2 \times R_{0 j} \times & \left(1-S_{u j}\right) \\
\leq & \bar{u}_{j} \leq H_{u j}-2 \times R_{0 j} \times\left(1-S_{u j}\right)
\end{aligned}
$$

where $S_{y i}$ and $S_{u j}$ are the percentage reduction in the base case variability of $y_{i}$ and $u_{j} . S_{y i}$ and $S_{u j}$ are obtained from MVPA. The inequalities defined for the problem allow $5 \%$ constraint limit violation.

Assessment of improved yield by relaxing constraints Relaxing the limits for the constraint variables can help move the quality variables closer to the optimum operating points, thus improving the yield. The optimization problem for this case is to solve (2) subject to (3)-(5) and (14)-(15)

$$
\begin{aligned}
& L_{y i}+2 \times \sigma_{i 0}-y_{h o l i} \times r_{y i} \\
& \leq \bar{y}_{i} \leq H_{y i}-2 \times \sigma_{i 0}+y_{h o l i} \times r_{y i} \\
& L_{u j}+2 \times R_{j 0}-u_{h o l j} \times r_{u j} \\
& \leq \bar{u}_{j} \leq H_{u j}-2 \times R_{j 0}+u_{h o l j} \times r_{u j}
\end{aligned}
$$

where $r_{y i}$ and $r_{u j}$ are the user specified percentage relaxation in the limits for $y_{i}$ and $u_{j}$. Again, the inequalities defined for the problem allow $5 \%$ constraint limit violation.

Constraint/variablity tuning for a desired yield Given a desired benefit, which CVs/MVs should be tuned? How much do they need to be tuned to achieve the given desired 
benefit? In particular, to which CV or MV is the economic benefit potential most sensitive? That is, which process variable does contribute the most in terms of the economic profit improvement? The problem of finding sensitive or profitable process variables to the economic profit is of prime importance in process control engineering and plant optimization. For a selective constraint/variability tuning guideline, see a sensitivity analysis in Lee et al. [2007].

\section{INDUSTRIAL CASE STUDY OF HYDROGEN UNIT USING PATS}

PATS has been implemented in a process industry. To illustrate its features and industrial relevance, an industrial case study of a hydrogen unit is conducted for APC performance monitoring using the main components of PATS.

\subsection{Process model and data collection}

The hydrogen unit produces high purity hydrogen product by thermal cracking of natural gas and steam and supplies pure hydrogen to the naphtha hydrotreaters, the gas oil hydrotreaters, and the light gas oil hydrotreater in a bitumen process industry. The model has $27 \mathrm{CVs}, 5 \mathrm{MVs}$, and 13 DVs. The input variable is actually either SP or OP, which is available for data collection. For notational simplicity, we denote input variables as MVs in APC performance assessment.

The process data collected for the APC performance analysis includes process input/output variables, constraint related parameters such as low/high limits and soft low/high limits, and optimization parameters such as linear/quadratic coefficients, etc. The data collection lasted for around 2 days with 1 min. sampling. Process data analysis will be conducted for the APC performance assessment and economic benefit improvement.

\subsection{APC application subject to constraints}

A multivariable model predictive controller has been implemented on the hydrogen unit. It has been designed to minimize operational costs and $\mathrm{H}_{2}$ production waste and maximize export steam. This is mainly achieved by running at a lower steam to carbon ratio and lower excess $\mathrm{O}_{2}$. The control objective of the MPC application and the linear/quadratic coefficients in the economic objective function are shown in Table 1, where CV4 is reformer flue gas $\mathrm{O}_{2}$ content, $\mathrm{CV} 8$ is export steam flow rate, MV2 is steam to carbon ratio, and MV3 is combined outlet temperature. Steam to carbon ratio and reformer furnace combustion excess $\mathrm{O}_{2}$ are minimized to optimize reformer combustion efficiency. Export steam flow is maximized subject to constraints. With the control objectives, the multivariable controller optimizes the hydrogen production by adjusting the manipulated variables. The base case operation data of the hydrogen unit are shown in Fig. 4, where only quality variables (CV4 was in 'off' status and MV2 was in 'initialization' status) are shown due to the large range of variables. The input and output data were collected with 1 min. sampling time for 16 hours, i.e., 3rd 12:00 PM to 4th 4:00 AM. It is noted that all of the controlled variables and manipulated variables are
Table 1. Control objective and optimization parameters used in the economic objective function. (All data have been normalized.)

\begin{tabular}{cccc}
\hline \hline CV/MV & Control objective & \multicolumn{2}{c}{ Economic objective } \\
\cline { 3 - 4 } & & 0 & $b$ \\
\hline CV4 & Minimize & 0 & 0.5 \\
CV8 & Maximize & 0 & -0.1 \\
MV2 & Minimize & 0 & 1 \\
MV3 & Minimize & 0 & 0.1 \\
Others & Constrain & 0 & 0 \\
\hline \hline
\end{tabular}
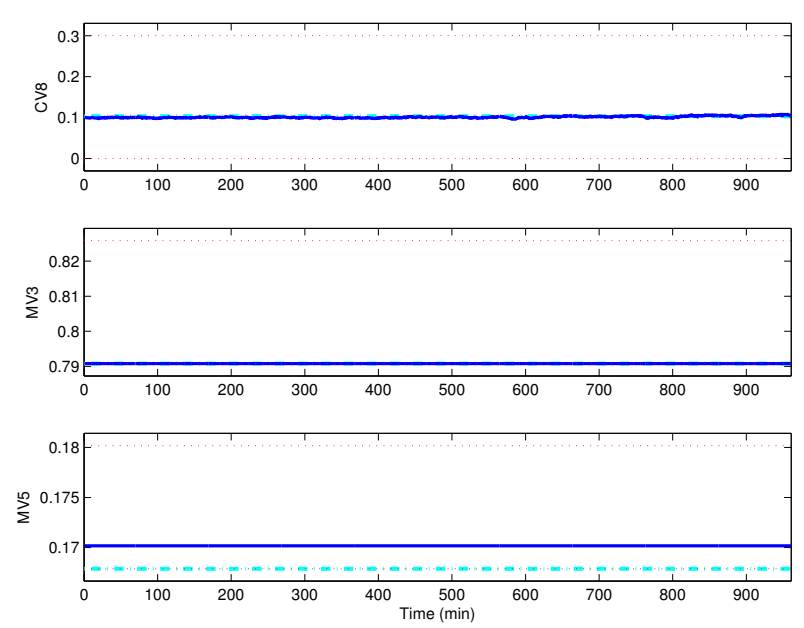

Fig. 4. Time series plot of quality variables CV8, MV3 and a constrained variable MV5 at the base case operation. Here, $-\cdot-$ shows the ideal operating point, which is discussed in Section 5.4.

operated within their constraints limits as shown in Fig. 4.

\subsection{Benefit potential analysis}

For performance assessment of the MPC application implemented in the hydrogen unit, the economic objective function is given by (1). The linear/quadratic coefficients are given in Table 1. Solving optimization problems specified in [Xu et al., 2007] for only input/output variables with 'on' status by using QP or SDP solvers, we calculate the economic performance costs $J_{0}=688.8811$ for the base case operation, $J_{I}=686.5121$ for the ideal operating condition, and $J_{E}=686.5121$ for the adjusted operating condition. Here, the process variables with 'off' status are not included in the optimization. Note that the ideal operation means the maximum benefit (or the minimum cost) that could be achieved if process variability could be reduced to zero. From the above performance assessment, it is noted that $J_{0}$ is very close to $J_{I}$ and $J_{E}$. It means that the hydrogen unit is operating close to its constraint limits. In addition, it is noted that the process variability of the hydrogen unit is seen to be very low. Hence it can be said that the MPC application is working well from the process variability point of view. It is also noted that $J_{E}$ turns into $J_{I}$ in this case because the variability of MV2 is almost zero and the ideal operating point of CV8 is located in the middle of its constraints.

The operating points for the ideal and base case operations are given in Table 2 , where $†$ indicates quality variables. It 
is noted that, as indicated by $*$ in Table 2 , for the variables MV3 and MV5, the ideal operating points are located at their low limits of the constraints.

\subsection{Selective constraint tuning guidelines}

As seen in the previous section, the MPC implemented on the hydrogen unit has good performance. Is there any further opportunity to improve the benefit? As discussed, process variability reduction would not bring additional benefit in this case. However, by finding opportunity to maximize CV8 and minimize MV3, we can improve benefit within an allowed limit. This can be done by relaxing the low or high limit of some process variables. Let us look at the location of the ideal operating point of the quality variables to provide a basis for constraint tuning. The ideal operating point of $\mathrm{CV} 8$ is located in the middle of the constraint, while the ideal operating point of MV3 is located at its low limit. Hence, it is observed that the limit change of CV8 itself will not help change its operating point. However, actually, there is a lot of room to maximize CV8 toward its high limit. From this observation, we will conduct three case studies to find a selective constraint tuning guideline that can be applicable to the running MPC application on the hydrogen unit. i) Find the most profitable process variable (PV). ii) Find the most contributing $\mathrm{PV}$ to maximize the operating point of CV8. iii) Find an alternative tuning guideline if the case studies 1-2 are not applicable.

To perform sensitivity analysis [Lee et al., 2007] for the case studies and suggest selective tuning guidelines, we calculate the benefit potentials. The ideal and existing benefit potentials are given by $\Delta J_{I}=2.369$ and $\Delta J_{E}=$ 2.369 , respectively. We assume that the desired benefit potential ratio is given by $R_{C}=1.2$. Note that, in this industrial application to the hydrogen unit, the desired benefit potential set as $\Delta J_{D}=2.8428$ is not an absolute target value to be achieved. It will be used to find a profitable PV or a suitable tuning guideline in constraint tuning.

Case study 1: Finding the most sensitive $P V$ To find the most sensitive or profitable PV, the constraint tuning optimization in [Lee et al., 2007] has been solved. From the optimization result, we find that the most profitable PV is MV3. Since MV3 is a quality variable and its control objective is to 'minimize' as shown in Table 1, the LL relaxation of MV3 according to the sensitivity analysis will guarantee benefit improvement. However, it is noticed that the constraint limit of MV3 has been kept without change due to the quality of the product. We will try to find the second sensitive PV in the next case study.

Case study 2: Maximizing the operating point of CV8 To find the second profitable PV, the constraint tuning optimization in [Lee et al., 2007] has been solved. From the optimization result, we find that the most profitable PV is MV5, which is not a quality variable. This implies that, although MV5 is not a quality variable, the LL relaxation of MV5 provides room to maximize the operating point CV8. MV5 is the most contributing $\mathrm{PV}$ to maximize CV8. What if the case studies 1-2 are not applicable to
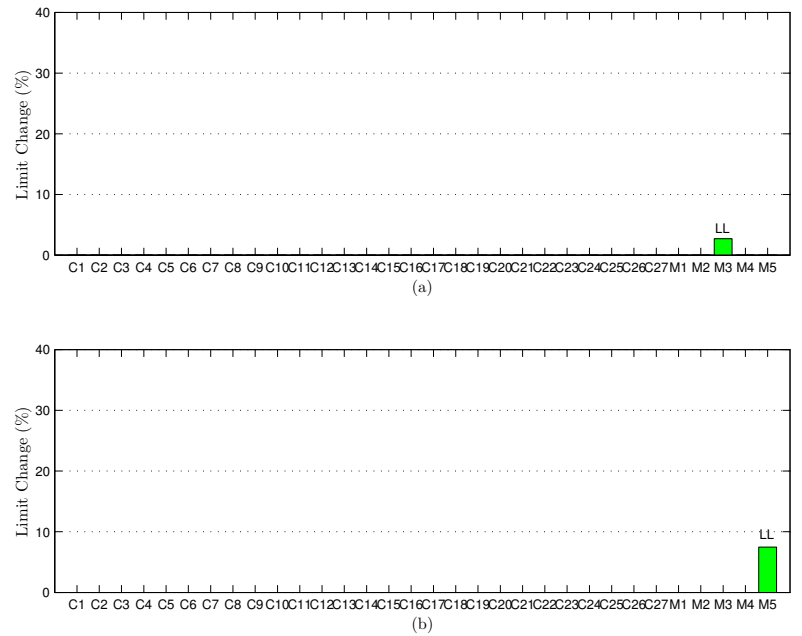

Fig. 5. Selective constraint tuning guidelines. (a) The case study 1. (b) The case study 2. 'LL' means the low limit change.

Table 2. The operating points for the ideal, the base case, and the case studies 1-3 operations. (All data have been normalized.)

\begin{tabular}{rrccc}
\hline \hline CV $/$ MV & Ideal & Base & CS-1 & CS-2 \\
\hline CV2 & 0.0012 & 0.0012 & 0.0012 & 0.0012 \\
CV3 & 0.2865 & 0.2865 & 0.2865 & 0.2865 \\
CV5 & 0.0125 & 0.0125 & 0.0125 & 0.0125 \\
CV6 & 0.9000 & 0.9000 & 0.8996 & 0.9000 \\
†CV8 & 0.1036 & 0.1012 & 0.1036 & $\mathbf{0 . 1 0 4 1}$ \\
CV9 & 0.3598 & 0.3685 & 0.3598 & 0.3580 \\
CV10 & 0.5109 & 0.5109 & 0.5105 & 0.5109 \\
CV12 & 0.4251 & 0.4251 & 0.4251 & 0.4251 \\
CV15 & 0.1197 & 0.1240 & 0.1197 & 0.1188 \\
CV16 & 0.2583 & 0.2583 & 0.2583 & 0.2583 \\
CV20 & 0.0499 & 0.0816 & 0.0499 & 0.0435 \\
CV22 & 0.0183 & 0.0183 & 0.0183 & 0.0183 \\
CV23 & 0.0981 & 0.0981 & 0.0981 & 0.0981 \\
CV24 & 0.2819 & 0.2819 & 0.2819 & 0.2819 \\
${ }^{\dagger}$ MV3 & $* 0.7908$ & 0.7908 & $\mathbf{0 . 7 9 0 3}$ & 0.7908 \\
MV5 & $* 0.1679$ & 0.1702 & 0.1679 & $\mathbf{0 . 1 6 7 4}$ \\
\hline \hline
\end{tabular}

the process unit. We will try to find an alternative for constraint tuning in the next case study.

Case study 3: What is an alternative tuning guideline?

To find an alternative to achieve the desired benefit potential, the constraint tuning optimization in [Lee et al., 2007] has been solved. From the optimization result, we observe that there is no feasible solution for this case study. This implies that we cannot improve the benefit without the LL change of MV3 or MV5 at this moment of the MPC performance assessment. MV3 and MV5 are the profitable PVs at this time. The constraint tuning guidelines obtained by the proposed sensitivity analysis for the case studies 1-2 are shown in Fig. 5. The operating points calculated for the case studies 1-2 are shown in Table 2 .

\subsection{Verification of economic benefit}

The data was collected for verification for 2 days, i.e., 4 th 1:00 AM to 6th 1:00 AM. The MV5 LL changed to 162 from 167.7 on 4 th. Fig. 6 shows the time series plot of 

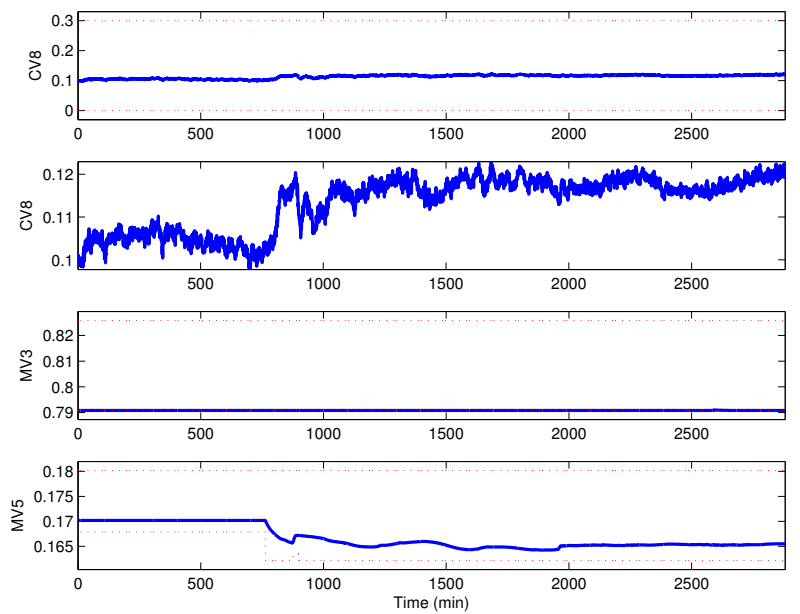

Fig. 6. Time series plot of quality variables CV8 and MV3 and a constrained variable MV5 at the MV5 LL change. The second figure is a closer look at CV8.
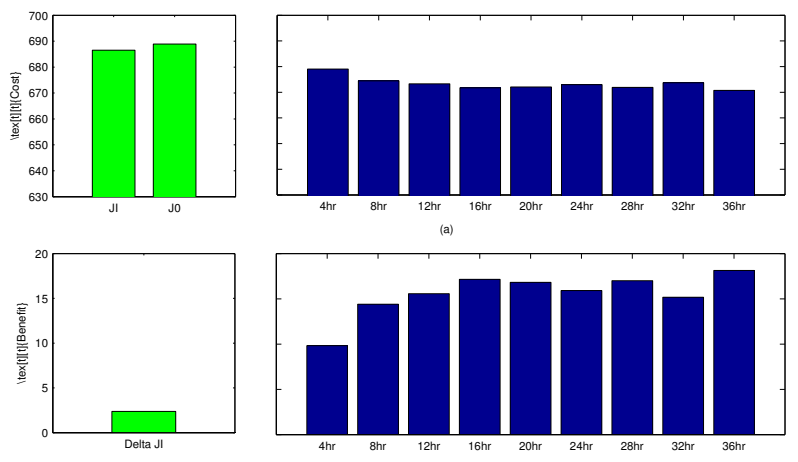

Fig. 7. (a) The comparison of cost. (b) The comparison of benefit. The left plots show $J_{I}, J_{0}$, and $\Delta J_{I}$ estimated in the performance assessment before the MV5 LL change. The right plots show $J_{0}$ and actual benefits calculated every 4 hours after the moment of the MV5 LL change.

quality variables at the moment of the MV5 LL change. As observed before in the case study 2 , it is actually shown that the MV5 LL relaxation maximizes the operating point of CV8, while it does not change the operating point of MV3. The computation of cost according to the MV5 LL change is shown in Fig. 7 . The right plot shows $J_{0}$ every 4 hours after the moment when the MV5 LL was changed. Note that the economic performance costs $J_{I}$ and $J_{0}$ for the ideal and base case operation before the MV5 LL change is $J_{I}=686.5121$ and $J_{0}=688.8811$, respectively. It is shown that $J_{0}$ is decreasing as CV8 is maximized by the MV5 LL change. To show the benefit improvement in terms of dollar value, we show a stewardship for the hydrogen unit. Fig. 8 shows the $\mathrm{H}_{2}$ production, the total $\mathrm{H}_{2}$ cost, and the savings in terms of normalized dollar value. As a consequence, it confirms that the constraint tuning significantly improves the profit of the process unit. The proposed sensitivity analysis provides a selective tuning guideline to find such an opportunity to improve the benefit in practice.
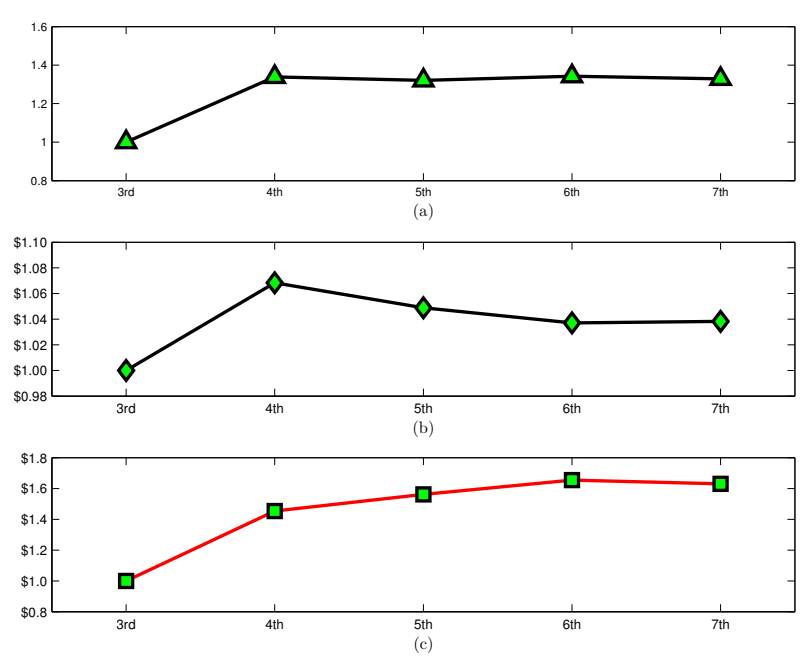

Fig. 8. Stewardship. (a) $\mathrm{H}_{2}$ production. (b) Total $\mathrm{H}_{2}$ cost. (c) Savings calculation. (All data have been normalized.)

\section{CONCLUSION}

In this paper, we have discussed the industrial implementation of advanced process control (APC) performance monitoring technology. A plant-oriented framework for industrial APC performance monitoring is proposed. A software package, called Performance Analysis Toolbox and Solutions (PATS), has been introduced, which integrates the APC performance analysis technologies for industry. The main components in PATS includes process data collection, data preprocessing, process model identification, similarity clustering, control valve stiction detection, multivariate controller performance assessment, and APC economic performance assessment using linear matrix inequality optimization. In particular, we have elaborated the last two major components for APC performance monitoring, which are referred to as MVPA and LMIPA, respectively. An industrial case study of a hydrogen unit is illustrated to show the applicability of the APC performance monitoring technology and the solutions package.

\section{REFERENCES}

B. Huang and S. L. Shah. Performance assessment of control loops: theory and applications. Spring-Verlag, London, 1999.

P. L. Latour, J. H. Sharpe, and M. C. Delaney. Estimating benefits from advanced control. ISA Transactions, 25 (4):13-21, 1986.

K. H. Lee, B. Huang, and E. C. Tamayo. Sensitivity analysis for selective constraint and variability tuning in performance assessment of industrial MPC. Accepted for Publication in Control Engineering Practice, 2007.

G. D. Martin, L. E. Turpin, and R. P. Cline. Estimating control function benefits. Hydrocarbon Processing, pages 68-73, 1991.

S. J. Qin and T. A. Badgwell. A survey of industrial model predictive control technology. Control Engineering Practice, 11:733-764, 2003.

F. Xu, B. Huang, and S. Akande. Performance assessment of model predictive control for variability and constraint tuning. Ind. Eng. Chem. Res., 46:1208-1219, 2007. 